published).

${ }^{3}$ H. Primakoff, Revs. Modern Phys. 31, 802 (1959)。 Our notation follows closely that of Primakoff. Equations from this reference are listed as Eq. (P- ); in this form, the formula referred to is Eq. (P-11a).

${ }^{4} \mathrm{~V}$. L. Telegdi, Phys. Rev. Letters $\underline{8}, 327$ (1962).

${ }^{5}$ We will use UFI to refer to the following set of assumptions ${ }^{3}$ : (1) $g_{A}$ and $g_{V}$ are the same for $\mu$ capture as for $\beta$ decay except for small form-factor corrections.

(2) The induced pseudoscalar coupling $g_{P}$ equals $8 g_{A}$ for $\mu$ capture. (3) The weak-magnetism coupling $g_{M}$ is given by the conserved vector current theory. All experiments on total or partial capture rates are primarily sensitive only to combinations of $G_{V}$ and $\Gamma_{A}$, where $G_{V}$ is the Fermi coupling constant (closely equal to $g_{V}$ ), and $\Gamma_{A}$, the Gamow-Teller coupling constant, is a combination of $g_{A}, g_{P}$, and $g_{M}$.

${ }^{6}$ This point has been stated previously without a convincing demonstration [L. Wolfenstein, Proceedings of the Tenth Annual International Rochester Conference on High-Energy Physics, 1960 (Interscience Publishers, Inc., New York, 1960), p. 529; H。A. Tolhoek, Nuclear Phys. 10, 606 (1959)].

${ }^{7} J$. R. Luyten, H. P. C. Rood, and H. A. Tolhoek (to be published) give this equation for the special case of closed-shell nuclei, for which $J_{a}=1$.

${ }^{8}$ Since no approximation has been made in Eq. (3) as to the value of $\bar{\nu}$, this result with $\Delta_{a}$ and $\delta_{a}$ as functions of $\bar{\nu}$ might be useful in evaluating the cross section for the inverse process in which neutrinos are incident on complex nuclei. See S. Berman, International Conference on Theoretical Aspects of High-Energy Phenomena, CERN Report 61-22, 1961 (unpublished).
${ }^{9} \mathrm{~K}$. Ford and J. Wills, Nuclear Phys. 35, 295 (1962); and private communication.

${ }^{10}$ If we assume $f(r)$ is different from zero only for $r<\bar{\nu}^{-1}$, then we obtain $\delta^{\prime} / \delta_{a} \simeq 1+\frac{1}{10} \bar{\nu}^{2} d^{2}$, where $\frac{3}{5} d^{2}$ is the mean-square radius of the distribution defined by $f(r)$. With the value of $d$ in reference $3, \delta^{\prime} / \delta_{a}=1.05$.

${ }^{11}$ This equation was first derived by $\mathrm{H}$. Primakoff in a private communication to one of us (L. W.).

${ }^{12}$ The rate of the reaction $\mu^{-}+C^{12} \rightarrow B^{12}+\nu[\mathrm{E}$. J. Maier, B. L. Bloch, R. M. Edelstein, and R. T. Siegel, Phys. Rev. Letters $\underline{6}, 417$ (1961); E. J. Maier, Ph.D.thesis, Carnegie Institute of Technology, 1962 (unpublished)] indicates that $\Gamma_{A}{ }^{2}$ agrees with the UFI value within about $25 \%$, while the rate of $\mu^{-}+\mathrm{He}^{3} \rightarrow \mathrm{H}^{3}+\nu$ [I. V. Falomkin et al. , Physics Letters 1 , 318 (1962); International Conference on High-Energy Nuclear Physics, Geneva, 1962 (CERN, Geneva, Switzerland, to be published)] gives a value of $G^{2}$ in agreement with UFI to better than $25 \%$. Recent measurements on muon capture in hydrogen [R. Hildebrand, Phys. Rev. Letters $\underline{8}, 34$ (1962); E. Bleser et al., Phys. Rev. Letters $\underline{8}, 288$ (1962); E. Bertolini et al. , International Conference on HighEnergy Nuclear Physics, Geneva, 1962 (CERN, Geneva, Switzerland, to be published)] are most likely consistent with UFI, although the rate appears somewhat low. If it is assumed that the axial-vector contributions $\left(g_{P}\right.$ and $g_{A}$ ) to $\mu$ capture are given by UFI, then both the $\mathrm{He}^{3}$ and hydrogen results require a vector contribution. If we relax the condition on the axial-vector contributions \{either because of some deviation from universality form-factor effects, or second-class current contributions [J。B. Adams, Phys. Rev. $126,1567(1962)]\}$, the analysis of present experiments does not exclude $g_{V}=0$.

\title{
CONSTRAINTS IMPOSED ON MANDELSTAM REPRESENTATION BY UNITARITY
}

\author{
Andre Martin* \\ University of Washington, Seattle, Washington and CERN, Geneva, Switzerland \\ (Received August 27, 1962; revised manuscript received October 8, 1962)
}

It is the hope of several physicists ${ }^{1}$ that by combining Mandelstam representation with unitarity one could determine completely a two-body scattering amplitude. Therefore, it seems important to reduce as much as possible the amount of information one has to put in a Mandelstam representation for a given process, to determine uniquely the corresponding scattering amplitude, by making use of unitarity. Much progress was made in this direction by Froissart ${ }^{2}$ who showed that in the Mandelstam representation with a finite number of subtractions,

$$
\begin{gathered}
F(s, t, u)=\frac{1}{\pi^{2}} s^{N} t^{N} \iint \frac{\rho_{u}\left(s^{\prime} t^{\prime}\right) d s^{\prime} d t^{\prime}}{\left(s^{\prime}-s\right)\left(t^{\prime}-t\right) s^{\prime} N_{t^{\prime}} N}+P_{s t u}+\sum_{p=0}^{M<N} t^{p} s^{M}-\int \frac{p, s}{\left(s^{\prime}\right) d s^{\prime}} \\
+\sum_{p q=0}^{L} t^{p} s^{q} \rho_{p q}+\text { pole terms }
\end{gathered}
$$

(where $P_{s t u}$ denotes a circular permutation), the subtraction terms, determined by $\rho_{p, s}$, etc., $\rho_{p q}$, 
could not be chosen completely arbitrarily once the double-spectral functions

$$
\rho_{u}(s, t), \rho_{t}(u, s), \rho_{s}(t, u)
$$

were given. His result, based on application of a weak form of unitarity to the high-energy behavior of a scattering amplitude, was that if two amplitudes $F$ and $F^{\prime}$ have in common the same double-spectral functions and the same pole terms, their difference consists, at the most, of a sum of six one-dimensional integrals and one constant, namely,

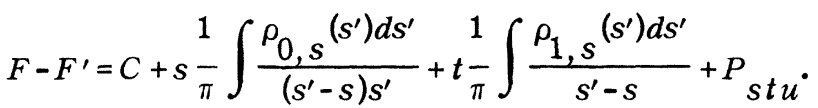

Here we want to improve this result by taking into account the existence of purely elastic regions in one or two channels in which the unitarity condition takes the form of an equation instead of an inequality. Our conclusions are the following:

(a) If in one channel, say $s$, there is a purely elastic region $s_{0}<s<s_{1}$, where $s_{1}$ can be chosen arbitrarily close to $s_{0}$, the knowledge of the double-spectral functions $\rho_{u}(s, t)$ and $\rho_{t}(u, s)$ in $s_{0}<s$ $<s_{1}$ determines completely the three double-spectral functions and the pole terms in the $u$ and $t$ channels. This is the case for $\pi N, \pi \pi$, or $N N$ scattering.

(b) If, in addition, there is a purely elastic region in a second channel, say $u$, the same amount of information determines the amplitude completely. This is the case for $\pi \pi$ and $\pi N$ scattering, but not for $N N$ scattering which is connected by cross ing to $N \bar{N}$ scattering only.

The amplitudes $F$ or $F^{\prime}$ describe reaction $A+B$ $\rightarrow A+\bar{B}$ ( $A$ and $B$ being spinless particles with masses $M_{A}$ and $M_{B}$ ) in the channel where $s$ is the square of the c.m. energy (channel I); channel II describes $A+\bar{B}-A+\bar{B}$, associated with $u$; channel III, associated with $t$, corresponds to $A+\bar{A} \rightarrow B+\bar{B}$. We have, of course, the standard relation $s+t+u=2 M_{A}^{2}+2 M_{B}{ }^{2} . \quad F$ satisfies representation (1), and $F^{\prime}$ satisfies an analogous representation where all the $\rho$ functions or coefficients have primed indices, and the pole terms may be different.

Our first assumption is that in channel $s$ there exists an elastic region, $s_{0}=\left(M_{A}+M_{B}\right)^{2}<s<s_{1}$, and that when $s$ lies in this region,

$$
\rho_{u}(s, t)=\rho_{u}{ }^{\prime}(s, t), \rho_{t}(u, s)=\rho_{t}^{\prime}(u, s) .
$$

Then by inspection of Eq. (1) one sees easily that the difference of the absorptive parts of $F$ and $F^{\prime}$ in channel $s, A$ and $A^{\prime}$, will be

$$
A(s, t)-A^{\prime}(s, t)=\sum_{p=0}^{M}\left[\rho_{p, s}(s)-\rho_{p, s}{ }^{\prime}(s)\right] t^{p} .
$$

This means that if we expand $F$ and $F^{\prime}$ in partial waves,

$$
\begin{gathered}
F=(\sqrt{ } / q s) \sum(2 l+1) f_{l}(s) P_{l}\left(\cos \theta_{s}\right), \\
F^{\prime}=(\sqrt{ } / q s) \sum(2 l+1) f_{l}^{\prime}(s) P_{l}\left(\cos \theta_{s}\right),
\end{gathered}
$$

where $q_{s}$ is the c.m. momentum in channel $s$ and $\theta_{S}$ the scattering angle, we have

$$
\operatorname{Im} f_{l}=\operatorname{Im} f_{l}^{\prime} \text { for } l>M \text {. }
$$

From the elastic unitarity condition, it follows that

$$
\left(\operatorname{Re} f_{l}\right)^{2}=\left(\operatorname{Re} f_{l}^{\prime}\right)^{2} \text { for } l>M .
$$

Now, it has been shown by many author $s^{3}$ that if $F$ satisfies the Mandelstam representation, then $f_{l}$ and also $\operatorname{Re} f_{l}$ can be interpolated by analytic functions of $l$, bounded by polynomials in $|l|$, for $\operatorname{Re} l>N$. More precisely, $\operatorname{Re} f_{l}$ can be interpolated by $\operatorname{Re} f_{l}^{+}$and $\operatorname{Re} f_{l}^{-}$, where $\operatorname{Re} f_{l}^{+}$ coincides with $\operatorname{Re} f_{l}$ for even integral values of $l$; according to Eq. (6) the analytic functions

$$
\left(\operatorname{Re} f_{l}^{+}-\operatorname{Re} f_{l}{ }^{+}\right)\left(\operatorname{Re} f_{l}^{+}+\operatorname{Re} f_{l}{ }^{++}\right)
$$

and

$$
\left(\operatorname{Re} f_{l}^{-}-\operatorname{Re} f_{l}^{\prime-}\right)\left(\operatorname{Re} f_{l}^{-}+\operatorname{Re} f_{l}^{\prime-}\right)
$$

vanish respectively for even and odd integral values of $l$ larger than $N$. From Carlson's theorem $^{4}$ it follows that they vanish identically for $\operatorname{Re} l>N$. Since each term of the two products is an analytic function of $l$, it follows that

$$
\left.\begin{array}{c}
\text { either } \operatorname{Re} f_{l}=\operatorname{Re} f_{l}^{\prime} \\
\text { or } \operatorname{Re} f_{l}=-\operatorname{Re} f_{l}^{\prime}
\end{array}\right\} \text { for all even } l>N \text {, }
$$

and

$$
\left.\begin{array}{c}
\text { either } \operatorname{Re} f_{l}=\operatorname{Re} f_{l}^{\prime} \\
\text { or } \operatorname{Re} f_{l}=-\operatorname{Re} f_{l}^{\prime}
\end{array}\right\} \text { for all odd } l>N \text {. }
$$

In other words, when the imaginary part of a purely elastic scattering amplitude is given, up 
to a polynomial in $\cos \theta_{s}$, there are only four possible real parts of the scattering amplitude (up to a polynomial in $\cos \theta_{S}$ ) which are consistent with the fixed-energy analytic properties implied by the Mandelstam representation.

Let us now show that the analytic properties of $F$ and $F^{\prime}$ with respect to $s$ force us to choose the solution

$$
\operatorname{Re} f_{l}=\operatorname{Re} f_{l}^{\prime} \text { for all } l>N .
$$

Let us first rule out the case

$$
\operatorname{Re} f_{l}=-\operatorname{Re} f_{l}^{\prime} \text { for all } l>N .
$$

For then one can easily see that the partial wave expansion of $F$ and $F^{\prime}$ can be re-summed and written as

$F+F^{\prime}=\frac{\sqrt{s}}{q s} \sum_{0}^{M}(2 l+1) \alpha_{l} P_{l}\left(\cos \theta_{s}\right)+2 i A(s, t)$.

Let us now evaluate the discontinuity of both sides over the positive $t$ cut. We find

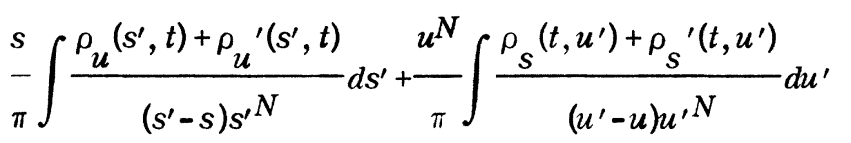

$$
+\sum_{0}^{M} c_{n}(t) s^{n}=2 i \rho_{u}(s, t)
$$

Now, it is a known property of the boundary curve of $\rho(s, t)$ that, given $t_{m}$ arbitrarily large, one can always find a region $s_{0}<s<s\left(t_{m}\right), 0<t$ $<t_{m}$, in which $\rho$ and $\rho^{\prime}$ vanish; this follows from application of the unitarity condition. Therefore, the right-hand side of the equation vanishes for $s_{0}<s<s\left(t_{m}\right)$ if $t$ is less than $t_{m}$. The left-hand side, however, is an analytic function of $s$ for fixed $t$ with two cuts which do not overlap over the region $s_{0}<s<s(t)$. It vanishes on a finite segment inside its analyticity domain and therefore vanishes identically. It follows that $\rho_{u}(s, t) \rho_{u}{ }^{\prime}(s, t)$ $\equiv 0$ for all $s$, and from assumption (3) that $\rho_{u}(s, t)$ $=0$ for $s_{0}<s<s_{1}$. This situation is not acceptable, and therefore the case $\operatorname{Re} f_{l}=-\operatorname{Re} f_{l^{\prime}}$ should be rejected.

The case $\operatorname{Re} f_{l}^{-}=\operatorname{Re} f_{l^{\prime-}}$ and $\operatorname{Re} f_{l}^{+}=-\operatorname{Re} f_{l}{ }^{\prime+}$ is more delicate, because then one has to work with amplitudes symmetrized with respect to $\cos \theta_{S}$ in order to eliminate the odd- $l$ part. However, the same type of argument works since the boundary curve of $\rho_{t}(s, u)$ has the same behavior as that of $\rho_{u}(s, t)$. In the simple case, $M_{A}=M_{B}$, the sym- metrized amplitude is just

$$
\frac{1}{2}[F(s, t, u)+F(s, u, t)],
$$

and one arrives at the equation

$$
\rho_{u}(s, t)+\rho_{t}(s, t)=0,
$$

valid in the elastic strip. Then the absorptive parts of the symmetrized amplitudes,

$$
\frac{1}{2}[F(s, t, u)+F(s, u, t)] \text { and } \frac{1}{2}\left[F^{\prime}(s, t, u)+F^{\prime}(s, u, t)\right] \text {, }
$$

are simply polynomials in $t$. Therefore, for $l>N$, $\operatorname{Im} f_{l}^{+}=\operatorname{Im} f_{l^{\prime+}}=0$ and also, from unitarity, $\operatorname{Re} f_{l}^{+}$ $=\operatorname{Re} f_{l}{ }^{++}=0$ for $l>N$. Hence, combining this with $\operatorname{Re} f_{l}{ }^{-}=\operatorname{Re} f_{l^{\prime-}}$ for $l>N$, we see that $F-F^{\prime}$ is a polynomial of degree $N$ in $t$ with coefficients depending on $s$. The proof still holds for $M_{A} \neq M_{B}$, but the writing is less easy because of the lack of symmetry between $t$ and $u$.

The case $\operatorname{Re} f_{l}^{-}=-\operatorname{Re} f_{l^{\prime-}}$ and $\operatorname{Re} f_{l}^{+}=\operatorname{Re} f_{l^{\prime+}}$ can be treated in a similar way.

In the end we see that for $l>N$ we have $\operatorname{Re} f_{l}$ $=\operatorname{Re} f_{l^{\prime}}$, for both odd and even $l$ 's, and hence

$$
F(s, t, u)-F^{\prime}(s, t, u)=\sum_{0}^{N} \alpha_{n}(s) t^{n} .
$$

Let us now evaluate the discontinuity of both sides of (8) over the $t>0$ cut. We find

$$
\begin{gathered}
-\int_{\pi}^{N} \frac{\rho_{u}\left(s^{\prime}, t\right)-\rho_{u}{ }^{\prime}\left(s^{\prime}, t\right)}{\left(s^{\prime}-s\right) s^{\prime} N} d s^{\prime}+-\frac{u^{N}}{\pi} \frac{\rho_{s}\left(t, u^{\prime}\right)-\rho_{s}{ }^{\prime}\left(t, u^{\prime}\right)}{\left(u^{\prime}-u\right) u^{\prime} N} d u^{\prime} \\
+\sum_{p=0}^{M} u^{p}\left[\rho_{p, t}(t)-\rho_{p, t}(t)\right]=0 .
\end{gathered}
$$

Here again the left-hand side of this equation is an anlytic function of $s$ with two cuts which do not overlap the region $s_{0}<s<s_{1}$ because of assumption (3). Therefore, since it vanishes inside its analyticity domain, it vanishes identically, and

$\rho_{u}(s, t) \equiv \rho_{u}{ }^{\prime}(s, t), \quad \rho_{s}(t, u) \equiv \rho_{s}{ }^{\prime}(t, u), \quad \rho_{p, t}(t) \equiv \rho_{p, t}{ }^{\prime}($

Similarly, by considering the discontinuity of (8) across the $u>0$ cut, we find

$$
\rho_{t}(u, s) \equiv \rho_{t}^{\prime}(u, s), \quad \rho_{p, u}(u) \equiv \rho_{p, u^{\prime}}(u) .
$$

Using these conditions to calculate the difference $F-F^{\prime}$, we discover that, in fact, formula (8) holds not only in the elastic strip, but everywhere. For 
convenience we rewrite it as

$$
F-F^{\prime}=\sum_{0}^{N} \beta_{n}(s) u^{n}
$$

Let us now consider the high-energy region of channel $u, u \rightarrow \infty$, and $s<0$. Channel $u$ is a scattering channel in which the Froissart limit ${ }^{2}$ $|F(s, t, u)|<c u \ln ^{2} u$ applies. Therefore, for $s<0$, $\beta_{n}(s)=0$ for $n>1$. Since this holds over a finite interval inside the analyticity domain of $F-F^{\prime}$ with respect to $s$, this holds everywhere. So

$$
F(s, t, u)-F^{\prime}(s, t, u)=\beta_{0}(s)+u \beta_{1}(s) .
$$

So only the $S$ and $P$ waves in channel $s$ may change when one replaces $F$ by $F^{\prime}$. Taking into account the unitarity condition, $\left|f_{0}\right|<1,\left|f_{1}\right|<1$, $\left|f_{0}{ }^{\prime}\right|<1,\left|f_{1}^{\prime}\right|<1$, we get, for large $s$,

$$
\beta_{0}(s)<\text { const, } \beta_{1}(s)<\text { const } \times s^{-1} \text {. }
$$

These conditions enable us to write

$$
\begin{aligned}
F-F^{\prime}= & C+\sum \frac{C_{i}}{s-s_{i}}+\frac{s}{\pi} \int_{s_{0}}^{\infty} \frac{w_{0}\left(s^{\prime}\right) d s^{\prime}}{s^{\prime}\left(s^{\prime}-s\right)}+u\left[\sum \frac{C_{i}{ }^{\prime}}{s-s_{i}{ }^{\prime}}\right. \\
& \left.+\int_{s_{0}}^{\infty} \frac{w_{1}\left(s^{\prime}\right) d s^{\prime}}{s^{\prime}-s}\right] .
\end{aligned}
$$

So, under assumption (3), the difference of $F$ and $F^{\prime}$ consists at most of one constant, two one-dimensional integrals determined by two spectral functions, and $S$ - and $P$-wave poles in the channel $s$. This means, in particular, that the three double-spectral functions of $F$ coincide with the three double-spectral functions of $F^{\prime}$, and that the poles of $F$ and $F^{\prime}$ in the $t$ and $u$ channels have the same positions and residues.

To go further, a new assumption is necessary. This will be that in another channel there exists a purely elastic region, say the $u$ channel. This will be the case for $\pi N$ scattering or $\pi \pi$ scattering, disregarding for the moment the difficulties associated with charge exchange scattering. This will not be possible for $N N$ scattering, because real $N \bar{N}$ scattering is always associated with anhiliation reactions. Then if such an assumption is valid, we get

$$
F-F^{\prime}=\gamma_{0}(u)+s \gamma_{1}(u),
$$

and, comparing with (9),

$$
\beta_{0}(s)=A+B s, \quad \beta_{1}(s)=C+D s .
$$

However, the unitarity requirements (10) im- pose $B=C=D=0$. Now, since $F$ and $F^{\prime}$ have a common domain of reality, $A$ has to be a real constant. Therefore, in the $s$ channel,

$$
\operatorname{Im} f_{0}=\operatorname{Im} f_{0}^{\prime} \text { and } \operatorname{Re} f_{0}= \pm \operatorname{Re} f_{0}^{\prime}
$$

in the elastic region. The case $\operatorname{Re} f_{0}=\operatorname{Re} f_{0}^{\prime}$ gives $F=F^{\prime}$. The case $\operatorname{Re} f_{0}=-\operatorname{Re} f_{0}^{\prime}$ gives

$$
\operatorname{Re} f_{0}=\left(q_{s} / \sqrt{s}\right) \times \text { const. }
$$

So, unless the $S$ wave of $F$ has this particular form, $F$ has to be identical to $F^{\prime}$. This form of the $S$ wave is very peculiar anyway, because it has no left-hand cut and, by analytic continuation, leaves no room for inelastic processes. So we can safely draw the conclusion that $F^{\prime}$, in general, has to be identical with $F$.

We have, in this last section, disregarded the difficulties associated with change exchange scattering. These difficulties do not occur in the first channel $s$, because one can always work in a pure isospin state. But then in the channel $u$, one has a mixture of isospin states. However, in most cases this difficulty may be overcome. Let us consider, for instance, $\pi^{+} \pi^{+}$scattering in channel $s$. This is a pure $I=2$ state and, knowing the weight function $\rho_{u}(s, t)$ for $4 \mu^{2}<s<s_{1}$, where $\mu$ is the pion mass, one knows $\rho_{t}(u, s)$ in the same domain by using Bose statistics symmetry. Therefore, $F-F^{\prime}=\beta_{0}(s)$, the $P$ wave being excluded. In channel $u$ we have a mixture of $I$ $=0,1,2$, states. However, $F(s, t, u)-F(t, s, u)$ contains only an $I=1$ contribution and the argument works. Hence,

$$
\beta_{0}(s)-\beta_{0}(t) \equiv \gamma_{0}(u)+s \gamma_{1}(u) .
$$

It can be seen readily that the only solution of this equation is $\beta_{0}(s)=A+B s+C s^{2}$ and by unitarity, $\beta_{0}(s)=A$, so that, in the end, $F$ is completely determined.

So far as we can see, there are two implications of this result. First, the fact that the subtraction terms do not contain any new physical information is not inconsistent with the idea that if the analytic continuation of the partial wave amplitudes with respect to the angular momentum $l$ can be extended from $\operatorname{Re} l>N$ to $\operatorname{Re} l<N$, this continuation correctly determines the full amplitude. This would not be true if some of the subtraction terms were independent parameters.

The second remark is that when one combines the present result with crossing symmetry one expects to get very strong conditions on the spectral function in the elastic strip of channel $s$. For 
instance, in neutral $\pi \pi$ scattering the knowledge of $\rho_{u}(s, t)$ in the elastic $s$ strip determines $\rho_{s}(t, u)$ in a way which, a priori, does not guarantee the crossing symmetry relation $\rho_{u}(x, y)=\rho_{s}(x, y)$. So, it may be either that $\rho_{s}$ is completely or almost completely determined by these constraints or that $\rho_{s}$ cannot fulfill these requirements at all. So either the analyticity unitarity program can be carried out very far, or it is inherently inconsistent.

It is a pleasure to acknowledge fruitful discus sions with Professor R. J. Eden, Professor T. Kibble, and Professor E. Lomon, and to thank Professor R. Geballe, Professor B. Jacobsohn, and Professor E. Henley for their kind hospitali- ty at the Summer Institute for Theoretical Physics of the University of Washington.

*Permanent address: CERN, Geneva.

${ }^{1} \mathrm{G}$. F. Chew, invited talk at the La Jolla Conference, 1961 (unpublished).

${ }^{2}$ M. Froissart, Phys. Rev。 123,1053 (1961).

${ }^{3}$ V. N. Gribov J. Exptl. Theoret Phys. (U.S.S. R.) 41, 1962 (1962) [translation: Soviet Phys. -JETP 14, 1395 (1962)]; A. Martin, Physics Letters 1, 72 (1962); E. J. Squires, Nuovo cimento 25,242 (1962); G. Prosperi, Nuovo cimento 24, 957 (1962); S. Okubo, preprint NYO 10322; R. Omnès, Nuovo cimento $\underline{25}, 806$ (1962).

'See, for instance, E. C. Titchmarsh, Theory of Functions (Oxford University Press, New York, 1939), p. 186.

NEUTRON FORM FACTORS AND NUCLEON

STRUCTURE. C. de Vries, R. Hofstadter, and Robert Herman [Phys. Rev. Letters 8 , 381 (1962)].

Besides other typographical errors indicated in a recent Erratum, ${ }^{1}$ we wish to point out that in Table I the value of $v_{2}$ is incorrectly given as 1.10. The quantity $v_{2}$ should have the value 1.15 . The corresponding value of $a_{2 p^{2}}{ }^{2} a_{2 n}{ }^{2}$ should be 1.57 fermi $\mathrm{m}^{2}$ instead of $1.50 \mathrm{fermi}^{2}$. No conclusions, data, or figures are altered by these corrections.

${ }^{1}$ C. de Vries, R. Hofstadter, and R. Herman, Phys. Rev. Letters $\underline{8}, 466(\mathrm{E})$ (1962). 